\title{
Ischemic Stroke Caused by Carotid Stump at the Common Carotid Artery
}

\author{
Go Hashimoto ${ }^{1}$, Shinichi Wada ${ }^{1}$, Takao Morita ${ }^{1}$, Saori Tomohara ${ }^{1}$, Kenta Hara ${ }^{2}$, \\ Mitsuru Kumabe ${ }^{3}$, Takumi Matsushima ${ }^{4}$, Masanori Kadowaki ${ }^{4}$, Masumitsu Hamaguchi ${ }^{5}$, \\ Takahiro Kuwashiro ${ }^{1}$, Masahiro Yasaka ${ }^{1}$ and Yasushi Okada ${ }^{1}$
}

\begin{abstract}
:
An 84-year-old man developed motor aphasia and right hemiparesis on postoperative day 1 after orchiectomy for suspected malignant lymphoma. He had a history of thoracic endovascular aortic repair for aortic aneurysm using a bypass graft from the right subclavian artery to the left common carotid artery (CCA); however, the graft had become occluded six months later. Brain magnetic resonance imaging revealed acute cerebral infarctions in the left frontal lobe. Carotid ultrasonography revealed a stump at the left CCA, just below the bifurcation, formed by the occluded graft with an oscillating thrombus. This case was rare in that a CCA stump was identified as the embolic source of ischemic stroke.
\end{abstract}

Key words: ischemic stroke, carotid stump syndrome, common carotid artery, ultrasonography

(Intern Med 59: 3071-3074, 2020)

(DOI: 10.2169/internalmedicine.5021-20)

\section{Introduction}

Carotid stump syndrome (CSS) is a cause of ischemic stroke due to thrombus forming at the site of an arterial stump (1). Most CSS cases are caused by thrombus at an internal carotid artery (ICA) stump, although it can also very occasionally form at a common carotid artery (CCA) stump $(2,3)$.

We herein report a case of ischemic stroke that occurred in a patient with a CCA stump diagnosed by ultrasonography.

\section{Case Report}

An 84-year-old man who had a history of thoracic endovascular aortic repair (TEVAR) with a bypass graft from the right subclavian artery to the left CCA for an aortic aneu- rysm 7 years earlier underwent orchiectomy for suspected malignant lymphoma on postoperative day (POD) 0. Computed tomography (CT) before the orchiectomy showed swelling of the testis and suspected lymphadenopathy in the para-aorta, mesentery, right iliac region and right inguinal canal. Although complete occlusion of the bypass graft had been identified by contrast CT six months after TEVAR, he had been medicated with warfarin and clopidogrel for seven years, and these antithrombotic drugs were discontinued two weeks before the orchiectomy procedure.

$\mathrm{He}$ developed motor aphasia and right hemiparesis on POD 1. His blood pressure was $145 / 74 \mathrm{mmHg}$, and he weighed $64.5 \mathrm{~kg}$. Blood findings of electrolytes and his hepatic function were unremarkable, but creatinine was 1.72 $\mathrm{mg} / \mathrm{dL}$, and coagulation tests showed high plasma D-dimer $(9.3 \mu \mathrm{g} / \mathrm{dL}$, Table). Brain magnetic resonance imaging (MRI) revealed a fresh ischemic lesion at the left middle cerebral artery territory that appeared as high- and low-

${ }^{1}$ Division of Cerebrovascular Medicine and Neurology, Clinical Research Institute, National Hospitalization Organization, Kyushu Medical Center, Japan, ${ }^{2}$ Division of Neuroendovascular Therapy, Clinical Research Institute, National Hospitalization Organization, Kyushu Medical Center, Japan, ${ }^{3}$ Department of Clinical Education and Training Center, National Hospitalization Organization, Kyushu Medical Center, Japan, ${ }^{4}$ Department of Hematology, Clinical Research Institute, National Hospitalization Organization, Kyushu Medical Center, Japan and ${ }^{5}$ Department of Urology, Clinical Research Institute, National Hospitalization Organization, Kyushu Medical Center, Japan

Received: April 7, 2020; Accepted: May 20, 2020; Advance Publication by J-STAGE: July 28, 2020

Correspondence to Dr. Shinichi Wada, ichi_basstrombone@yahoo.co.jp 
Table. Blood Examination Findings on Stroke Onset.

\begin{tabular}{cclr}
\hline Hematology & & ALT & $44 \mathrm{U} / \mathrm{L}$ \\
Leukocytes & $13,700 / \mu \mathrm{L}$ & BUN & $27 \mathrm{mg} / \mathrm{dL}$ \\
Erythrocytes & $389 \times 10^{6} / \mu \mathrm{L}$ & Creatinine & $1.72 \mathrm{mg} / \mathrm{dL}$ \\
Hemoglobin & $11.9 \mathrm{~g} / \mathrm{dL}$ & Sodium & $134 \mathrm{mEq} / \mathrm{L}$ \\
Hematocrit & $32.9 \%$ & Potassium & $4.5 \mathrm{mEq} / \mathrm{L}$ \\
Platelets & $26.9 \times 10^{3} / \mu \mathrm{L}$ & Cloride & $98 \mathrm{mEq} / \mathrm{L}$ \\
Coagulation & & T-chol & $104 \mathrm{mg} / \mathrm{dL}$ \\
PT-INR & 1.10 & LDL-chol & $48 \mathrm{mg} / \mathrm{dL}$ \\
APTT & $28.1 \mathrm{sec}$. & HDL-chol & $36 \mathrm{mg} / \mathrm{dL}$ \\
D-dimer & $9.3 \mu \mathrm{g} / \mathrm{mL}$ & Glucose & $93 \mathrm{mg} / \mathrm{dL}$ \\
Biochemistry & & HbAlc (NGSP) & $5.7 \%$ \\
Total protein & $6.8 \mathrm{~g} / \mathrm{dL}$ & C-reactive protein & $3.83 \mathrm{mg} / \mathrm{dL}$ \\
Albumin & $3.7 \mathrm{~g} / \mathrm{dL}$ & BNP & $32.6 \mathrm{pg} / \mathrm{mL}$ \\
AST & $51 \mathrm{U} / \mathrm{L}$ & Soluble interleukin-2 recepter & $3,537 \mathrm{U} / \mathrm{mL}$ \\
\hline
\end{tabular}

PT-INR: international normalized ration for prothrombin time, APTT: activated partial thromboplastin time, AST: aspartate aminotransferase, ALT: alanine aminotransferase, BUN: blood urea nitrogen, T-chol: total cholesterol, LDL-chol: low density lipoprotein cholesterol, HDL-chol: high density lipoprotein cholesterol, HbA1c (NGSP): hemoglobin A1c (National Glycohemoglobin Standardization Program), BNP: brain natriuretic peptide

intensity signals on diffusion-weighted and apparent diffusion coefficient images, respectively (Figure A). Cervical CT angiography showed the left CCA and left subclavian artery ligated by TEVAR and complete occlusion of the bypass graft connecting the right subclavian artery to the left CCA and left subclavian artery. Thus, the stump was formed at distal side of the left CCA, just below the bifurcation (Figure $\mathrm{B}, \mathrm{C}$ ).

Four-dimensional (4-D) contrast CT indicated blood flow stasis at the stump of the left CCA (Figure D). The length of the stump from the bifurcation was $19 \mathrm{~mm}$. The left ICA mainly flowed from the external carotid artery via the right superficial temporal artery (Figure E-G). Ultrasonography revealed a stump with oscillating thrombus and spontaneous echo contrast (SEC) at the distal side of the left CCA (Figure H). A Holter electrocardiography (ECG), transthoracic echocardiography and transesophageal echocardiography did not reveal any other significant embolic sources. We therefore diagnosed him with CSS caused by the stump at the CCA and treated the patient with warfarin and clopidogrel.

The thrombus detected at the time of the stroke onset gradually extended with central echolucency on POD 4 (Figure I). Finally, he was diagnosed with diffuse large Bcell lymphoma (germinal center B-cell-like phenotype, Ann Arbor stage IV, International Prognostic Index: highintermediate risk). Thus, he was treated with chemotherapy (cyclophosphamide, doxorubicin and prednisone: CHP) from POD 12. Stroke did not recur after the index stroke following the administration of warfarin and clopidogrel. However, ischemic stroke recurred in the left frontal lobe on POD 42 when warfarin was less effective (prothrombin timeinternational normalized ratio: 1.58). Ultrasonography on POD 42 revealed a new low-echoic thrombus even though the oscillating thrombus had stabilized (Figure J). Adding heparin erased the low-echoic thrombus on POD 45 without further ischemic stroke recurrence (Figure K). The length of the stump from the bifurcation (2-way arrow) was $10 \mathrm{~mm}$ on POD 45 (Figure L). However, motor aphasia and severe right hemiparesis persisted. The lymphadenopathy increased on $\mathrm{CT}$, and the malignant lymphoma was unresponsive to chemotherapy unfortunately. Although additional chemotherapy (rituximab, cyclophosphamide, doxorubicin, vincristine and prednisone: R-CHOP) was administered, the lymphadenopathy did not decrease on CT on POD 49. Considering his age and activity of daily living (performance status: 4, modified Rankin Scale: 5), he was transferred to a palliative care hospital on POD 61.

\section{Discussion}

Ischemic stroke due to CSS is caused by thrombus that forms at the stump of an occluded vessel. Most CSS cases are caused by thrombus at an ICA stump $(2,3)$. The mechanism is thought to involve a thrombus at the ICA stump forming due to large plaque flows into the intracranial ICA via collateral circulation from the external carotid artery (4). Our patient was unique in that ischemic stroke was induced by thrombus that formed at the CCA stump after a bypass vessel became occluded and anticoagulant therapy was discontinued. In addition, lymphoma may have induced thrombus formation due to hypercoagulation, since tumor cells and stimulation of tumor-associated inflammatory cells activate the clotting cascade (5).

Only one case report has described CSS caused by a CCA stump that formed due to chronic occlusion of the CCA along with a thrombus detected by carotid ultrasonography synchronously oscillating with the cardiac cycle (6). Just as in that case, an oscillating thrombus with high echo was also found at the onset of ischemic stroke in our patient. Furthermore, the thrombus extended gradually 

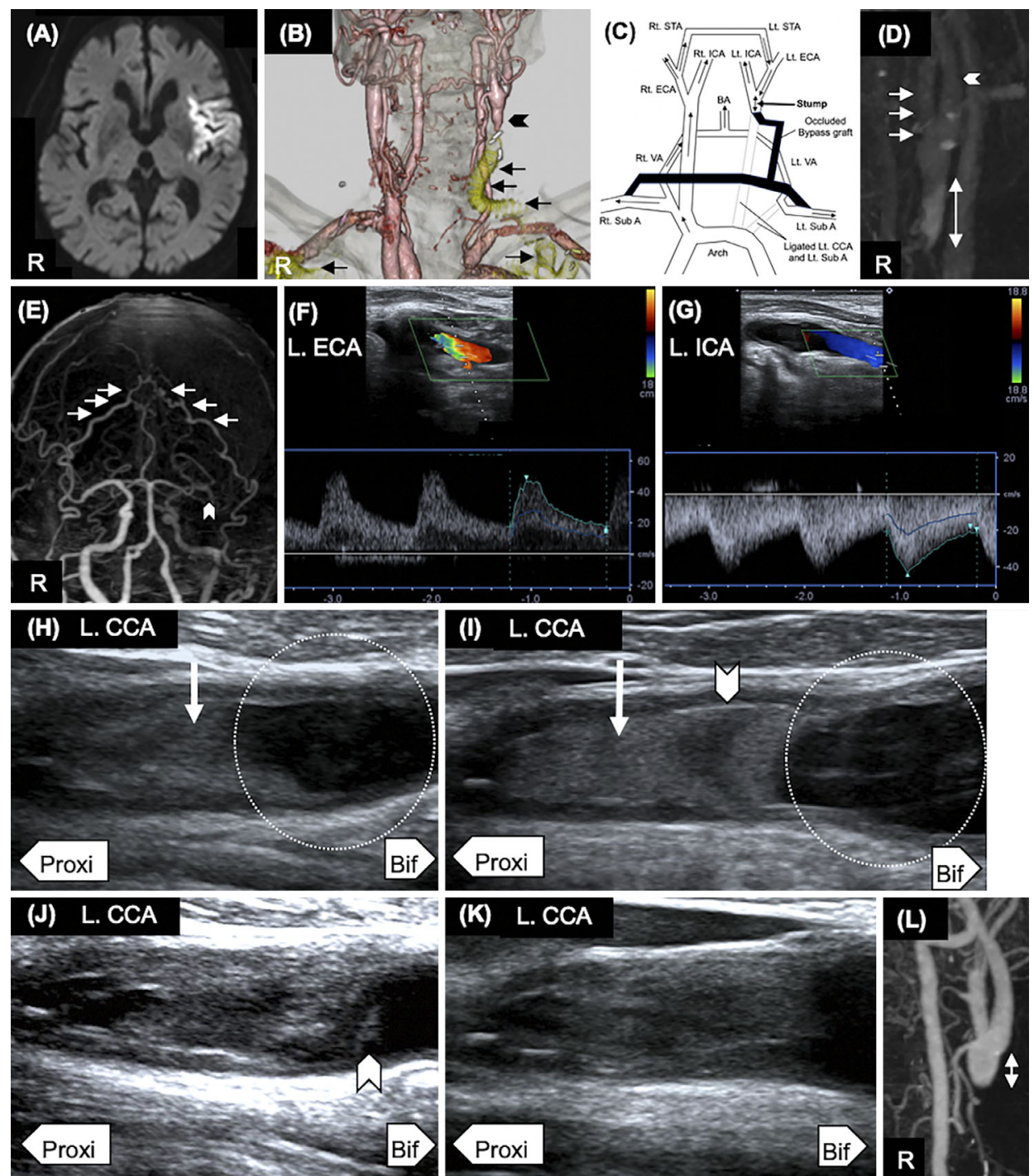

Figure. Imaging findings. (A) Diffusion-weighted magnetic resonance imaging showed high-intensity lesions in the left middle cerebral artery (POD1). R: right side. (B) By total arch replacement, the origin of the left common carotid artery (CCA) and left subclavian artery was ligated. Cervical CT angiography (POD 1) showed complete occlusion of the bypass graft connecting the right subclavian artery to the left CCA and left subclavian artery (arrow). Thus, a stump was formed at the distal side of the left CCA (arrowhead). (C) Schematic illustration of the arterial hemodynamics based on cervical contrast $\mathrm{CT}$ in this patient. Rt: right, Lt: left, Sub A: subclavian artery, VA: vertebral artery, ECA: external carotid artery, STA: superficial temporal artery, ICA: internal carotid artery, BA: basilar artery, CCA: common carotid artery. (D) Cervical four-dimensional (4-D) contrast CT (POD 1) showed a stump at the distal side of the left CCA. Blood flow stasis was observed at the stump of the left CCA in the venous phase. The length of the stump at the CCA from the bifurcation (2-way arrow) was $19 \mathrm{~mm}$. The arrow indicates the left external carotid artery (ECA), and the arrowhead indicates the left internal carotid artery (ICA). (E) Head 4-D contrast CT (POD 2) showed that the left ECA flow was mainly supplied from the left superficial temporal artery (STA) via the right STA (D). In addition, the left middle cerebral artery was occluded (arrowhead), which caused ischemic stroke. (F) The flow of the left ECA (L. ECA) was retrograde according to carotid ultrasonography (CU) on POD 1. (G) The flow of the left ICA (L. ICA) was anterograde according to CU on POD 1. (H) Oscillating thrombus (arrow) and spontaneous echo contrast (SEC, circle) were detected by CU on POD 1. Proxi: proximal side, Bif: bifurcation side. (I) The thrombus with oscillating thrombus (arrow) detected at the time of the stroke onset was gradually extended with central echolucency (arrowhead) on POD 4. SEC was still observed (circle). (J) Ultrasonography on POD 42 revealed a new low-echoic thrombus (arrowhead) even though the oscillating thrombus had stabilized. (K) The low-echoic thrombus had disappeared by POD 42. (L) The length of the stump from the bifurcation (2-way arrow) was $10 \mathrm{~mm}$ on POD 45. 
with central echolucency, which was the low-echoic portion, thus indicating that liquid rapidly formed within the thrombus (7). This shift from low- to high-echoic content stabilized the thrombus and reduced the risk of recurrent ischemic stroke (8).

Our patient also had SEC, which is blood flow with a slowly moving, cloud-like swirling pattern of "smoke" that is considered to indicate a risk of thrombus $(9,10)$. This is a rouleaux formation caused by low shear rates and is associated with the formation of consecutive local thrombi (9). Indeed, a new, low-echoic thrombus formed at the site of SEC when warfarin was ineffective in our patient. Warfarin does not affect SEC, although hematocrit, fibrinogen and other factors do, so SEC persisted despite the administration of antithrombotic therapy (10).

The treatment for CSS is antithrombotic agents, surgical ligature of the stump or endovascular therapy with stenting (11). However, the priority of surgery or endovascular therapy compared with medication has not been fully investigated. Both surgery and endovascular therapy were considered difficult to perform in the present patient because of the complex vascular structure or focal adhesions due to stasis after bypass-graft surgery. Total anticoagulation therapy might be needed for secondary prevention even after confirming that a thrombus has stabilized.

We described a patient with ischemic stroke caused by rare thrombus formation at the stump of the CCA. CSS should be considered when other embolic sources are undetectable and a stump is evident.

The authors state that they have no Conflict of Interest (COI).

\section{References}

1. Quill DS, Colgan MP, Sumner DS. Carotid stump syndrome: a colour-coded doppler flow study. Eur J Vasc Surg 3: 79-83, 1989.

2. Zhang XU, Shao S, Zheng X, Gao X, Zhang Y. Carotid stump syndrome: a case report. Exp Ther Med 10: 1161-1164, 2015.

3. Cassidy L, Grace PA, Bouchier-Hayes DJ. The carotid stump syndrome. Eur J Vasc Surg 6: 368-370, 1992.

4. Lakshminarayan R, Scott PM, Robinson GJ, Ettles DF. Carotid stump syndrome: pathophysiology and endovascular treatment options. Cardiovasc Intervent Radiol 34 (Suppl 2): S48-S52, 2011.

5. Liu B, Li B, Zhou P, et al. Prognostic value of pretreatment plasma D-dimer levels in patients with diffuse large B cell lymphoma (DLBCL). Clin Chim Acta 482: 191-198, 2018.

6. Omoto S, Hasegawa Y, Sakai K, et al. Common carotid artery stump syndrome due to mobile thrombus detected by carotid duplex ultrasonography. J Stroke Cerebrovasc Dis 25: e205-e207, 2016.

7. Haugland JM, Asinger RW, Mikell FL, Elsperger J, Hodges M. Embolic potential of left ventricular thrombi detected by twodimensional echocardiography. Circulation 70: 588-598, 1984.

8. Meissner MH, Moneta G, Burnand K, et al. The hemodynamics and diagnosis of venous disease. J Vasc Surg 46: 4S-24S, 2007.

9. Kawabori M, Yoshimoto T, Ito M, et al. Spontaneous echo contrast and thrombus formation at the carotid bifurcation after carotid endarterectomy. Neurol Med Chir (Tokyo) 52: 885-891, 2012.

10. Ito T, Suwa M, Nakamura T, Miyazaki S, Hirota Y, Kawamura K. Influence of Warfarin therapy on left atrial spontaneous echo contrast in nonvalvular atrial fibrillation. Am J Cardiol 84: 857-859, 1999.

11. Hrbac T, Benes V, Sirucek P, et al. Safety and efficacy of surgical treatment of carotid stump syndrome: pilot study. Ann Vasc Surg 26: $797-801,2012$

The Internal Medicine is an Open Access journal distributed under the Creative Commons Attribution-NonCommercial-NoDerivatives 4.0 International License. To view the details of this license, please visit (https://creativecommons.org/licenses/ by-nc-nd/4.0/).

(C) 2020 The Japanese Society of Internal Medicine Intern Med 59: 3071-3074, 2020 